\section{Kompass \\ Dermatologie}

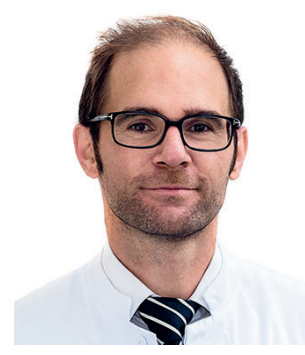

\section{Andreas Körber}

Gemeinschaftspraxis Dr. Cesko \&

Prof. Dr. Körber, Essen, Deutschland

Liebe Leserin, lieber Leser,

ich wünsche Ihnen ein frohes und lebendiges neues Jahr. Wir haben für Sie in unseren Ausgaben im Jahr 2020 einen breit gefächerten Strauß an Themen vorbereitet, welche die Dermatologie in den nächsten Jahren maßgebend beeinflussen werden. Den Auftakt macht in der ersten Ausgabe die Versorgungsforschung. Die Bedeutung der Versorgungsforschung ist im letzten Jahrzehnt ungemein gestiegen. Herr Professor Augustin, welcher freundlicher- (und glücklicher-)weise als Gasteditor dieser Ausgabe fungiert, hat wesentlich dazu beigetragen, dass die Versorgungsforschung heutzutage aus der täglichen Therapieentscheidung nicht mehr wegzudenken ist. Wir haben gelernt, Qualitäten zu messen und zu berücksichtigen. Durch Daten aus Registern wie dem PsoBest Register oder aus einer Vielzahl von Studien zum Patientennutzen von Therapien haben wir heute die Möglichkeit, unsere Entscheidungen unter Berücksichtigung von weit mehr als nur reinen Effektivitätsdaten zu treffen. Dies wird schon alleinig bei der Betrach-

\title{
Herzlich willkommen zum Kompass Dermatologie 2020
}

tung der atopischen Dermatitis augenscheinlich. Es geht darum, uns weiter stets zu verbessern.

Die operative Dermatologie ist eine der bedeutendsten Säulen der Dermatologie. Neben der Tumorchirurgie spielt sie beispielsweise bei der Hidradenitis suppurativa eine bedeutsame Rolle. Auch die operative Dermatologie steht im Fokus sich ändernder Therapieprinzipien. Neue Methoden wie die Photooptoakustik oder die Möglichkeiten der virtuellen Realität, etwa das Einblenden von Schnittbildern in das OP-Gebiet, sind faszinierende Techniken, die vor der Tür stehen.

Sicherlich gab es wenige Themen, die zuletzt die Dermatologie so dominiert haben, wie die Dermatoonkologie. Diagnostische und therapeutische Meilensteine gab es sowohl beim Melanom als auch beim Merkelzellkarzinom und diese haben die Prognosen der Tumoren signifikant beeinflusst.

Abschließend werden wir in der letzten Ausgabe 2020 einen Blick in die Zukunft unseres Fachs werfen. Digitale Medizin ist in aller Munde und wird auch die Dermatolo- gie nachhaltig verändern. Neben praktischen Anwendungen, wie der Diagnostik von melanozytären Hautveränderungen, wird sie Einzug finden in die Histologie, in die Kommunikation an Sektorgrenzen wie der Klinik/Praxis Schnittstelle und auch in den (Dokumentations-) Alltag.

Wir dürfen sehr gespannt sein auf ein neues Jahr, neue Erkenntnisse, müssen uns aber auch an ein gleichbleibend hohes, wenn nicht sogar rasantes Tempo gewöhnen, mit denen Neuerungen ihren Weg in den dermatologischen Alltag finden.

Ich hoffe, ich habe Ihr Interesse geweckt und wünschen Ihnen viel Freude bei allen Ausgaben des Kompass Dermatologie 2020. Mit herzlichen Grüßen Ihr

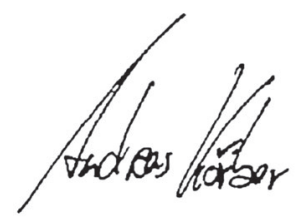

Andreas Körber 